\title{
A REPRESENTATION OF THE SOLUTIONS OF THE DARBOUX EQUATION IN ODD-DIMENSIONAL SPACES
}

\author{
BY \\ H. RHEE( ${ }^{(1)}$
}

\begin{abstract}
It is shown that determining a function from its averages over all spheres passing through the origin leads to an explicit representation of the even solutions of the Darboux equation in the exterior of the characteristic cones in terms of the hyperboloidal means of the boundary data on the cones.
\end{abstract}

The purpose of this paper is to derive a representation of the even-solutions of the Darboux equation $\sum_{i=1}^{n} \partial^{2} V / \partial x_{i}^{2}-(n-1) / t \partial V / \partial t-\partial^{2} V / \partial t^{2}=0$ in the exterior of the characteristic cones $C_{0}\left(t= \pm|X|, X \in R^{n}, n\right.$ odd $\left.\geqq 3\right)$ in terms of its prescribed values on $C_{0}$. Let $f(X)$ be a continuous function defined in $R^{n}$. The spherical means, SM, of $f$ is defined as follows:

$$
\operatorname{SM}[f ; X, \rho]=\omega_{n}^{-1} \int_{\alpha} f(X+\rho \alpha) d \omega_{\alpha},
$$

where $X$ is the center of the sphere of radius $\rho . \alpha$ denotes a unit vector. When $\rho=|X|$, we put $\mathrm{SM}[f ; X,|X|]=\mathrm{SM}^{*} f$.

The main part of this paper is concerned with the problem of determining functions from their integrals over a family of spheres passing through the origin. Specifically, if $J(X)$ is a given function in $R^{n}$, the main problem is to find $f(X)$ which satisfies

$$
J(X)=\mathrm{SM}^{*} f .
$$

We shall show that inverting (1) is closely related to the representation of the evensolutions of the Darboux equation. The problem of determining functions from their integrals over spheres of a fixed radius was studied by F. John [3].

In this paper we shall make use of the means values of functions defined in $R^{n}$ over the confocal hyperboloids which have the origin $O$ and a point $X=r \alpha$, $r=|X| \neq 0$, as foci, and the line $\overline{O X}$ as the axis of rotational symmetry. The

Received by the editors August 19, 1969 and, in revised form, November 20, 1969.

AMS Subject Classifications. Primary 3506, 3579.

Key Words and Phrases. Darboux equation, characteristic cones, spherical means, hyperboloidal means, John-Asgeiersson identity, Radon transform.

(1) This work was partially supported by NSF Grant GP-5087. It is part of a doctoral thesis written under the direction of Professor Y. W. Chen and presented to the University of Massachusetts. The author would like to thank Professor Chen for his guidance and encouragement. 
hyperboloid can be described in terms of its intersection with any plane passing through the axis of symmetry. If $(\sigma, \tau)$ are the coordinates in such a plane with $(\sigma, \tau)=(0,0)$ at the origin and $(\sigma, \tau)=(r, 0)$ at the point $X$, then the family of hyperbolas with parameter $t$ is given in $(\sigma, \tau)$ coordinates by

$$
t^{-2}(\sigma-r / 2)^{2}-\left(r^{2}-t^{2}\right)^{-1} \tau^{2}=1 / 4
$$

with $t$ in $0 \leqq|t|<r$. Note that when $t=0$, the hyperboloid is the plane bisecting $\overline{O X}$. From (2) it follows that $t$ means geometrically the differences of distances from the foci. A point $Y=\rho \gamma, \rho=|Y|$, will lie on the hyperboloid of parameter $|t|$, if

$$
\gamma=p \alpha+p^{\prime} \alpha^{\prime}
$$

where $\alpha^{\prime}$ is a unit vector perpendicular to $\alpha$,

$$
p=\left(2 t \rho+r^{2}-t^{2}\right) / 2 r \rho, \quad p^{\prime}=\left(1-p^{2}\right)^{1 / 2}, \quad|t|<r, \quad(r+t) / 2 \leqq \rho<\infty .
$$

The point $Y$ will be on one of the two sheets of the hyperboloids, depending on whether $t=|t|$ or $t=-|t|$. We denote the two sheets by $h(x, t)$ and $h(x,-t)$. Let $F_{1}$ be the family of all those circles which have their centers on the hyperbola (2) and pass through the origin. Then $F_{1}$ has as envelope the circle $c((r, 0),|t|)$ with center at $(\sigma, \tau)=(r, 0)$ and with radius $|t|$. If $(\sigma, \tau)$ is any point in the exterior of $c((r, 0),|t|)$, there are two circles of $F_{1}$ passing through $(\sigma, \tau)$.

Let $J(X)$ be a continuous function in $R^{n}-0$ (the origin deleted). The hyperboloidal means, HM, of $J$ over the sheet $h(x, t)$ is defined as follows:

$$
\mathrm{HM}\left[|Y|^{n-3} J(Y) ; X, t\right]=\left(\omega_{n-1} r^{n-2}\right)^{-1} \int_{c}^{\infty} d \rho \int_{\alpha^{\prime}} \rho^{n-3} J(\rho \gamma)\left(1-p^{2}\right)^{(n-3) / 2} d \omega_{\alpha^{\prime}},
$$

where $\gamma$ is given by (3), $c=(r+t) / 2$.

A function $f(X)$ defined in $R^{n}-0$ (or $R^{n}$ ) is said to belong to the class $C_{\varepsilon}$, $0<\varepsilon<1$, if $f(X)$ is continuous and $f(X)=O\left(|X|^{-n+2-\varepsilon}\right)$ for large $|X|$. The HM of $J$ exists, if $J \in C_{\varepsilon}$. It is easily verified that if $f \in C_{\varepsilon}$, then $\mathrm{SM}^{*} f \in C_{\varepsilon}$.

Theorem 1. Let $f \in C_{\varepsilon}$ in $R^{n}$ and $\psi(X)=\mathrm{SM}^{*} f$. For $X=r \alpha \neq 0$ and $0<t<r<\infty$, let

$$
U(X, t)=\mathrm{HM}\left[|Y|^{n-3} \psi(Y) ; X, t\right]+\operatorname{HM}\left[|Y|^{n-3} \psi(Y) ; X,-t\right] .
$$

Then the following identity holds for $0<|t|<r$,

$$
\mathrm{SM}[f ; X, t]=\left(c_{1} t\right)^{-1}\left(r^{2} N\right)^{(3-n) / 2}\left[T\left(-r^{2} / t \cdot\right)\right]^{(n-3) / 2} \operatorname{Tr}^{n-2} U(X, t),
$$

where $T=\left(r^{2}+t^{2}\right) / 2 r \partial / \partial t+t \partial / \partial r, N=\left(r^{2}-t^{2}\right) / r, c_{1}=-((n-3) / 2) !$.

(4) with $n=3$ was derived in [1]. (4) expresses the SM of $f$ in terms of the hyperboloidal means of $\mathrm{SM}^{*} f$ in the exterior of the cones $C_{0}$. It therefore follows from (4) that we will have a representation for the even-solutions of the Darboux equation in the exterior of $C_{0}$ in terms of prescribed values on $C_{0}$, if the equation (1) can be inverted. The problem of inverting (1) will be considered in Theorem 3. 
In the proof of Theorem 1 we shall make use of the following well-known identity on iterated spherical means by John and Asgeiersson [2],

$$
\int_{\zeta} d \omega_{\zeta} \int_{\eta} F(s \eta+r \zeta) d \omega_{\eta}=2 \omega_{n-1} \int_{|r-s|}^{r+s} J \tau d \tau \int_{\zeta} F(\tau \zeta) d \omega_{\zeta}
$$

where $J=\left\{\left[(r+s)^{2}-\tau^{2}\right]\left[\tau^{2}-(r-s)^{2}\right]\right\}^{(n-3) / 2}(2 r s)^{-n+2}$.

Proof of Theorem 1. Let $X=r \alpha \neq 0$ be a point in $R^{n}$ and let $\beta$ and $\gamma$ be two unit vectors in $R^{n}$. We may write

$$
\beta=q I+q^{\prime} \beta^{\prime}, \quad \gamma=p I+p^{\prime} \gamma^{\prime},
$$

where $I$ is the unit vector on the first axis, $\beta^{\prime}$ and $\gamma^{\prime}$ are two unit vectors in $R^{n-1}$, $p^{\prime}=\left(1-p^{2}\right)^{1 / 2}, q^{\prime}=\left(1-q^{2}\right)^{1 / 2}$. It suffices for our proof to take $X=(r, 0,0, \ldots, 0)$ and $\alpha=(1,0,0, \ldots, 0)$. By letting $d \omega_{\beta}=\left(1-q^{2}\right)^{(n-3) / 2} d q d \omega_{\beta^{\prime}}$ and applying (6), we obtain

$$
\begin{aligned}
\mathrm{SM}^{*} f & =\omega_{n}^{-1} \int_{\beta} f(\rho(\gamma+\beta)) d \omega_{\beta} \\
& =\omega_{n}^{-1} \int_{-1}^{1}\left(1-q^{2}\right)^{(n-3) / 2} d q \int_{\beta^{\prime}} f\left(\rho(p+q) I+\rho\left(p^{\prime} \gamma^{\prime}+q^{\prime} \beta^{\prime}\right)\right) d \omega_{\beta^{\prime}} .
\end{aligned}
$$

Using (5) and (7), we obtain

$\mathrm{HM}\left[|Y|^{n-3} \psi(Y) ; X, t\right]$

$$
=2 c_{2} r^{-n+2} \int_{c}^{\infty} \rho^{n-3} d \rho \int_{-1}^{1}\left(1-q^{2}\right)^{(n-3) / 2} d q \int_{|\lambda-\mu|}^{\lambda+\mu}\left(1-p^{2}\right)^{(n-3) / 2} P F \tau d \tau,
$$

where $\lambda=\rho\left(1-p^{2}\right)^{1 / 2}, \mu=\rho\left(1-q^{2}\right)^{1 / 2}, c_{2}=\omega_{n-2}\left(\omega_{n} \omega_{n-1}\right)^{-1}$,

$$
\begin{aligned}
& P=\left\{\left[(\lambda+\mu)^{2}-\tau^{2}\right]\left[\tau^{2}-(\lambda-\mu)^{2}\right]\right\}^{(n-4) / 2}(2 \lambda \mu)^{-n+3}, \\
& F=\int_{\eta} f(\rho(p+q) I+\tau \eta) d \omega_{\eta} .
\end{aligned}
$$

If we let $P^{\prime}=\left\{\left[(\lambda+\mu)^{2}-\tau^{2}\right]\left[\tau^{2}-(\lambda-\mu)^{2}\right]\right\}^{(n-4) / 2}$ and $\sigma=\rho(p+q)$, (8) may be written as

$$
2^{-n+4} c_{2} r^{-n+2} \int_{c}^{\infty} \rho^{-n+2} d \rho \iint_{G_{\rho}} P^{\prime} F \tau d \tau d \sigma,
$$

where the domain of integration $G_{\rho}$ in the $(\sigma, \tau)$-plane is obtained as follows: Let $e(\rho)$ and $e^{\prime}(\rho)$ be circles of the family $F_{1}$ with their centers on $h(x, t)$, and let the points $\left(\rho p, \rho p^{\prime}\right)$ and $\left(\rho p,-\rho p^{\prime}\right)$ be the centers of $e(\rho)$ and $e^{\prime}(\rho)$ respectively. If $e$ and $e^{\prime}$ denote the disks bounded by $e(\rho)$ and $e^{\prime}(\rho)$, then $G_{\rho}=e-\left(e \cap e^{\prime}\right)$. If we now replace $\lambda$ and $\mu$ by $\rho p^{\prime}$ and $\rho q^{\prime}$ respectively, we have

$$
\begin{aligned}
\left\{\left[(\lambda+\mu)^{2}-\tau^{2}\right]\left[\tau^{2}-(\lambda-\mu)^{2}\right]\right\}^{(n-4) / 2} & =\left(-A-2 B \rho-C \rho^{2}\right)^{(n-4) / 2} \\
& =\left[A \rho^{2}\left(b_{1}-1 / \rho\right)\left(1 / \rho-b_{2}\right)\right]^{(n-4) / 2},
\end{aligned}
$$


where $A=\left(\sigma^{2}+\tau^{2}\right)\left((\sigma-N)^{2}+\tau^{2}\right), \quad B=m(\sigma-N) \xi^{2}, \quad C=m^{2} \xi^{2}-4 \tau^{2}, \quad \xi^{2}=\sigma^{2}+\tau^{2}$, $m=2 t / r$. Since $G_{\rho}$ lies in the exterior of the circle $c((r, 0),|t|)$, we have $r\left(B^{2}-A C\right)$ $=4 \xi^{2} \tau^{2} N\left(\tau^{2}+(\sigma-r)^{2}-t^{2}\right)>0$ for each $(\sigma, \tau)$ in $G_{\rho}$. This justifies the decomposition of $\left(-A-2 B \rho-C \rho^{2}\right)$. Thus (8) becomes

(9) $\mathrm{HM}\left[|Y|^{n-3} \psi(Y) ; X, t\right]=2^{-n+4} c_{2} r^{-n+2} \int_{c}^{\infty} \rho^{-2} d \rho \iint_{G_{\rho}}(A Q)^{(n-4) / 2} F \tau d \tau d \sigma$, where $Q=\left(b_{1}-1 / \rho\right)\left(1 / \rho-b_{2}\right)$. Replacing $t$ by $-t$ in (9), we find

$$
\begin{aligned}
& \mathrm{HM}\left[|Y|^{n-3} \psi(Y) ; X,-t\right] \\
& \quad=2^{-n+4} c_{2} r^{-n+2} \int_{b}^{\infty} \rho^{-2} d \rho \iint_{G_{\rho}^{\prime}}\left(A Q^{\prime}\right)^{(n-4) / 2} F \tau d \tau d \sigma,
\end{aligned}
$$

where $b=(r-t) / 2, G_{\rho}^{\prime}=G_{\rho}(r,-t, \rho), Q^{\prime}=Q(r,-t, \sigma, \tau, \rho)$. In order to form the sum of (9) and (10) we introduce $\rho^{\prime}=-\rho$ in (10) as the new variable of integration. The intervals $c \leqq \rho<\infty$ in (9) and $-\infty<\rho^{\prime} \leqq-b$ will be disjoint. The result of replacing $t$ by $-t$ in $\left(A+2 B \rho+C \rho^{2}\right)$ is the same as changing $\rho$ to $\rho^{\prime}$, since of the three coefficients $A, B$ and $C$, only $B$ contains odd powers of $t$. Hence we have $Q^{\prime}=Q(r,-t, \sigma, \tau, \rho)=Q\left(r, t, \sigma, \tau, \rho^{\prime}\right)$ and $G_{\rho}^{\prime}=G_{\rho}\left(r, t, \rho^{\prime}\right)$. Since $\rho^{\prime}$ is now only a variable of integration, we may use $\rho$ instead of $\rho^{\prime}$ in (10). Therefore we may express the sum of (9) and (10) as

$$
U(X, t)=2^{-n+4} c_{2} r^{-n+2}\left\{\int_{c}^{\infty}+\int_{-\infty}^{-b}\right\} \rho^{-2} d \rho \iint_{G_{\rho}}(A Q)^{(n-4) / 2} F \tau d \tau d \sigma
$$

We shall next interchange the order of integrations of $d \rho$ and $d \tau d \sigma$ in (11). Note that in order for a point $(\sigma, \tau)$ to be in a domain $G_{\rho}$ it must lie in the exterior of the circle $c((r, 0),|t|)$, since $G_{\rho}$ lies in the exterior of $c((r, 0),|t|)$. We know that through each point in the exterior of $c((r, 0),|t|)$ there pass two circles of the family $F_{1}$. This means that $(\sigma, \tau)$ lies on the boundaries of two of the domains $G_{\rho}$. Now $(\sigma, \tau)$ can be in $G_{\rho}$ if and only if $b_{2}<1 / \rho<b_{1}$. Therefore it is clear that these domains are given by the value of $\rho$ with $b_{1}=1 / \rho$ and $b_{2}=1 / \rho$. Hence by keeping $(\sigma, \tau)$ fixed in the domain $K$ (the exterior of the circle $c((r, 0),|t|)$ with $\tau>0)$, we find that the limits of integration with respect to $1 / \rho$ are simply $b_{1}$ and $b_{2}$. Thus we may write (11) as

$$
\begin{aligned}
U(X, t)= & 2^{-n+4} c_{2} r^{-n+2} \iint_{K} A^{(n-4) / 2} F \tau d \tau d \sigma \\
& \cdot \int_{b_{2}}^{b_{1}}\left[\left(b_{1}-1 / \rho\right)\left(1 / \rho-b_{2}\right)\right]^{(n-4) / 2} d(1 / \rho) .
\end{aligned}
$$

It is easily seen that $b_{2}-b_{1}=2 A^{-1}\left(B^{2}-A C\right)^{1 / 2}$ and

$$
\int_{b_{2}}^{b_{1}}\left[\left(b_{1}-1 / \rho\right)\left(1 / \rho-b_{2}\right)\right]^{(n-4) / 2} d(1 / \rho)=c_{3} A^{-n+3}\left\{4 \xi^{2} \tau^{2} N r^{-1}\left(\tau^{2}+(\sigma-r)^{2}-t^{2}\right)\right\}^{(n-3) / 2},
$$


where $c_{3}=2^{n-3} \Gamma^{2}((n-2) / 2) \Gamma^{-1}(n-2)$. Hence (12) takes the form

$$
U(X, t)=c_{2} c_{3} r^{-n+2} \iint_{K} A^{(2-n) / 2} F\left(H^{1 / 2} \xi\right)^{n-3} \tau^{n-2} d \tau d \sigma,
$$

where $H=N r^{-1}\left(\tau^{2}+(\sigma-r)^{2}-t^{2}\right)$. Next apply the operator $T\left(r^{n-2} \cdot\right)$ to (13). In order to differentiate on the limits of integration we write

$$
\iint_{K} G d \tau d \sigma=\left\{\int_{0}^{r-t} d \sigma \int_{0}^{\infty}+\int_{r+t}^{\infty} d \sigma \int_{0}^{\infty}+\int_{r-t}^{r+t} d \sigma \int_{l}^{\infty}\right\} G d \tau,
$$

where $G=A^{(2-n) / 2} F\left(H^{1 / 2} \xi\right)^{n-3} \tau^{n-2}, l=\left(t^{2}-(\sigma-r)^{2}\right)^{1 / 2}$. We observe that differentiation on the limits of integration does not contribute anything, since the integrand vanishes when $\tau=l$. Note that $T N=0$ and $T A=0$. Hence we have

$$
\begin{aligned}
& N^{-1} T\left(r^{n-2} U(X, t)\right) \\
& =c_{2} c_{3} r^{-n+2}(n-3) / 2\left(-t / r^{2}\right) \iint_{K} A^{(2-n) / 2} F H^{(n-5) / 2} \xi^{n-1} \tau^{n-2} d \tau d \sigma .
\end{aligned}
$$

Since $2^{n-3} \omega_{n-2} \Gamma^{2}[(n-2) / 2] \omega_{n-1}^{-1} \Gamma^{-1}(n-2)=1$, by applying the operator $T\left(-r^{2} t^{-1} \cdot\right)$ to (14) we obtain

$$
\begin{aligned}
N^{-2} T( & \left.-r^{2} t^{-1} \cdot\right) T\left(r^{n-2} U(X, t)\right) \\
& =(n-5)(n-3) 2^{-1} \omega_{n}^{-1}\left(-t r^{-2}\right) \iint_{K} A^{(2-n) / 2} F H^{(n-7) / 2} \xi^{n+1} \tau^{n-2} d \tau d \sigma .
\end{aligned}
$$

If we continue applying $T\left(-r^{2} t^{-1} \cdot\right)$ to (15), we obtain

$$
\begin{aligned}
N^{(3-n) / 2}[ & \left.T\left(-r^{2} t^{-1} \cdot\right)\right]^{(n-5) / 2} \operatorname{Tr}^{n-2} U(X, t) \\
= & ((n-3) / 2) ! 2 \omega_{n}^{-1}\left(-t r^{-2}\right) \\
& \cdot\left\{\int_{0}^{r-t} d \sigma \int_{0}^{\infty}+\int_{r+t}^{\infty} d \sigma \int_{0}^{\infty}+\int_{r-t}^{r+t} d \sigma \int_{l}^{\infty}\right\} A^{(2-n) / 2} \xi^{2 n-6} \tau^{n-2} d \tau .
\end{aligned}
$$

Since $2 T l=\left(\sigma^{2}+l^{2}\right) t / r l, A^{(2-n) / 2}=\left[\left(\sigma^{2}+l^{2}\right) t r^{-1}\right]^{2-n}$ for $\tau=l$, and

$$
\omega_{n}^{-1} \int_{r-t}^{r+t} l^{n-3} \int_{\eta} f(\sigma I+l \eta) d \omega_{\eta} d \sigma=t^{n-3} \operatorname{SM}[f ; X, t]
$$

we finally obtain (4) by applying $T\left(-r^{2} t^{-1} \cdot\right)$ once more to (16).

In the next theorem we shall show that the solution of the Darboux equation given by (4) attains its prescribed values on the cones ' $C_{0}$. The left-hand side of (4) clearly goes to $\mathrm{SM}^{*} f$, as $t \rightarrow r$. But it is not obvious that the right-hand side of (4) also goes to $\mathrm{SM}^{*} f$, as $t \rightarrow r$. Note that we have the factor $r\left(r^{2}-t^{2}\right)^{-1}$ on the right-hand side.

TheOREM 2. Let $J(x)=\mathrm{SM}^{*} f$, and let $U(X, t)=\mathrm{HM}\left[|Y|^{n-3} J(Y) ; X, t\right]$ $+\mathrm{HM}\left[|Y|^{n-3} J(Y) ; X,-t\right]$. If we assume that $\delta^{i} J \in C_{\varepsilon}, \delta=\partial / \partial r r^{2} \cdot, 0 \leqq i \leqq$ $(n-1) / 2$, and let $t \rightarrow r$, then the right-hand side of $(4)$ reduces to $J(X)$. 
Proof. Since $p=1$ for $\rho=c$, and $p^{\prime}=1$ for $\rho=b$, we have

$T\left(r^{n-2} U(X, t)\right)$

$$
=\left(r \omega_{n-1}\right)^{-1}\left\{\int_{c}^{\infty} d \rho \int_{\alpha^{\prime}}\left(1-p^{2}\right)^{k} \delta J^{\prime} d \omega_{\alpha^{\prime}}-\int_{b}^{\infty} d \rho \int_{\alpha^{\prime}}\left(1-p^{\prime 2}\right)^{k} \delta J^{\prime} d \omega_{\alpha^{\prime}}\right\},
$$

where $k=(n-3) / 2, J^{\prime}=r^{n-3} J$. Applying $T\left(-r^{2} t^{-1} \cdot\right)$ to the above equation and observing that $T\left(-r^{2} t^{-1}\right)=\left(r^{2}-t^{2}\right) / 2 t^{2}$, we obtain

$$
\begin{aligned}
& T\left(-r^{2} t^{-1} \cdot\right) T^{n-2} U(X, t) \\
& =\omega_{n-1}^{-1}\left\{\left(r^{2}-t^{2}\right) / 2 t^{2} \int_{c}^{\infty} d \rho \int_{\alpha^{\prime}}\left(1-p^{2}\right)^{k} \delta J^{\prime} d \omega_{\alpha^{\prime}}\right. \\
& -t^{-1} \int_{c}^{\infty} d \rho \int_{\alpha^{\prime}}\left(1-p^{2}\right)^{k} \delta^{2} J^{\prime} d \omega_{\alpha^{\prime}} \\
& -\left(r^{2}-t^{2}\right) / 2 t^{2} \int_{b}^{\infty} d \rho \int_{\alpha^{\prime}}\left(1-p^{\prime 2}\right)^{k} \delta J^{\prime} d \omega_{\alpha^{\prime}} \\
& \left.-t^{-1} \int_{b}^{\infty} d \rho \int_{\alpha^{\prime}}\left(1-p^{\prime 2}\right)^{k} \delta^{2} J^{\prime} d \omega_{\alpha^{\prime}}\right\}
\end{aligned}
$$

It is easily seen that

$$
\begin{aligned}
& \left(1-p^{2}\right)=N\left(4 \rho^{2} r\right)^{-1}(2 \rho+(r-t))(2 \rho-(r+t)) \\
& \left(1-p^{\prime 2}\right)=N\left(4 \rho^{2} r\right)^{-1}(2 \rho+(r+t))(2 \rho-(r-t))
\end{aligned}
$$

If we apply $T\left(-r^{2} t^{-1} \cdot\right)$ to (17) $(k-1)$-times, use (18), (19), and let $t \rightarrow r$, then the right-hand side of (4) reduces to

$$
(k !)^{-1} r^{1-n-k}\left\{(-1)^{k+1} \int_{r}^{\infty} \rho^{-k}(\rho-r)^{k} \delta^{k+1} J^{\prime} d \rho+\int_{0}^{\infty} \rho^{-k}(\rho+r)^{k} \delta^{k+1} J^{\prime} d \rho\right\}
$$

Using regularity condition on $J$ at infinity and observing that when $t=r, \delta^{k+1} J^{\prime}$ is a function of $\rho$ only, we obtain $\int_{0}^{\infty} \rho^{-k}(\rho+r) \delta^{k+1} J^{\prime} d \rho=0$ and

$$
\int_{r}^{\infty} \rho^{-k}(\rho-r)^{k} \delta^{k+1} J^{\prime} d \rho=(-1)^{k+1} k ! r^{k+n-1} J(X)
$$

Hence (20) reduces to $J(X)$.

We now turn to the following problem: If $J(X)$ is a given function in $R^{n}$ ( $n$ odd $\geqq 3$ ), determine $f$ which satisfies $\mathrm{SM}^{*} f=J(X)$. It follows from (13) that

$$
U(X, 0)=2\left((n-1) \omega_{n}\right)^{-1} r^{-n+2} \Delta_{X} \iint|Y|^{-1}|Y-X| f(Y) d Y
$$


If we now assume that $f(X)$ satisfies a Hölder condition, we have

$$
r \Delta_{X}^{(n-1) / 2}\left(r^{n-2} U(X, 0)\right)=(-1)^{(n-1) / 2}(n-2) ! 2 f(X) .
$$

(21) can be written in the form

$$
f(X)=M r^{-2 n+3} \int_{r / 2}^{\infty} d \rho \int_{\alpha^{\prime}}\left(4-r^{2} / \rho^{2}\right)^{(n-3) / 2} \delta^{n-1}\left(\rho^{n-3} J\left(\rho \gamma_{0}\right)\right) d \omega_{\alpha^{\prime}},
$$

where $M=(-1)^{(n-1) / 2}\left[\omega_{n-2} \Gamma^{2}\left((n-2) 2^{-1}\right) 2^{n-5}(n-2)\right]^{-1}$,

$$
\gamma_{0}=X / 2 \rho+\left(1-r^{2} / 4 \rho^{2}\right)^{1 / 2} \alpha^{\prime} .
$$

Let $J(X)$ be a given function of class $C^{n-1}$ in $R^{n}$, such that

$$
\partial^{k} J / \partial r^{k}=O\left(|X|^{-2 n+3-k-\varepsilon}\right) \text { for large }|X|, \quad 0 \leqq k \leqq n-1,0<\varepsilon<1 .
$$

If $\mathrm{SM}^{*} f=J(X)$ has a solution $f \in C_{\varepsilon}$ with Hölder condition, then $f$ is uniquely given by (22). In order to show that the function $f$ given by (22) satisfies (1) we shall make use of the following Radon transform [2] and Lemma,

$$
(-1)^{(n-1) / 2} 2^{n} \pi^{n-1} f(Z)=\Delta_{Z}^{(n-1) / 2} \int_{\Omega_{X}} F(X, Z \cdot X) d \omega_{X},
$$

where $n$ is odd, $F(X, p)=\int_{Y \cdot X=p} f(Y) d S_{Y}$ and $|X|=1$. (The use of the Radon transform was suggested to me by Professor Y. W. Chen. It was shown in [4] without making use of the Radon transform that (22) is a solution of (1).)

LEMMA. When $n$ is odd $\geqq 3$,

$$
\mathrm{SM}^{*} r \Delta_{X}^{(n-1) / 2} r^{n-2} U(X, 0)=r \Delta_{X}^{(n-1) / 2} r^{n-2} \mathrm{SM}^{*} U(X, 0) .
$$

Proof. It is easily seen that

$$
\begin{aligned}
r \Delta_{X}^{(n-1) / 2} & r^{n-2} \Phi=\left[\prod_{k=1}^{(n-1) / 2}\left(r^{-n+2 k+2} \Delta_{X} r^{n-2 k}\right)\right] \Phi \\
r^{-n+2 k+2} \Delta_{X} r^{n-2 k} \Phi & =r^{-n+2 k+2}\left[\partial^{2} / \partial r^{2}+(n-1) / r \partial / \partial r+1 / r^{2} \Delta_{\alpha}\right] r^{n-2 k} \Phi \\
& =(2 n-2 k-2)(n-2 k) \Phi+(3 n-4 k-1) r \Phi_{r}+r^{2} \Phi_{r r}+\Delta_{\alpha} \Phi .
\end{aligned}
$$

Note that we may write

$$
\mathrm{SM}^{*} \Phi=2^{n-1} \omega_{n}^{-1} \int_{0}^{1} \lambda^{n-2}\left(1-\lambda^{2}\right)^{(n-3) / 2} d \lambda \int_{\alpha^{\prime}} \Phi(\rho \gamma) d \omega_{\alpha^{\prime}}
$$

where $\rho=2 r \lambda, \gamma=\lambda \alpha+\left(1-\lambda^{2}\right)^{1 / 2} \alpha^{\prime}$. Observing that $\Delta_{\alpha} \mathrm{SM}^{*} \Phi=\mathrm{SM}^{*} \Delta_{\gamma} \Phi, r \partial / \partial r=\rho$ $\partial / \partial \rho, r^{2} \partial^{2} / \partial r^{2}=\rho^{2} \partial^{2} / \partial \rho^{2}$, and using (27), we have

$$
\begin{aligned}
r^{-n+2 k+2} \Delta_{X} r^{n-2 k} \mathrm{SM}^{*} \Phi= & \mathrm{SM}^{*} \Delta_{\gamma} \Phi+\mathrm{SM}^{*}[(2 n-2 k-2)(n-2 k) \Phi] \\
& +\mathrm{SM}^{*}\left[(3 n-4 k-1) \rho \Phi_{\rho}\right]+\mathrm{SM}^{*}\left[\rho^{2} \Phi_{\rho \rho}\right] \\
= & \mathrm{SM}^{*}\left(r^{-n+2 k+2} \Delta_{X} r^{n-2 k} \Phi\right) .
\end{aligned}
$$


From (26), (28) it follows that

$$
\begin{aligned}
r \Delta_{X}^{(n-1) / 2} r^{n-2} \mathrm{SM}^{*} U(X, 0) & =\left[\prod_{k=1}^{(n-1) / 2}\left(r^{-n+2 k+2} \Delta_{X} r^{n-2 k}\right)\right] \mathrm{SM}^{*} U(X, 0) \\
& =\mathrm{SM}^{*}\left[\prod_{k=1}^{(n-1) / 2}\left(r^{-n+2 k+2} \Delta_{X} r^{n-2 k}\right)\right] U(X, 0) \\
& =\mathrm{SM}^{*} r \Delta_{X}^{(n-1) / 2} r^{n-2} U(X, 0) .
\end{aligned}
$$

THEOREM 3. Let $J(X)$ be a given function of class $C^{n-1}$ in $R^{n}$ with the property (23). Then the function $f(X)$ given by (21) satisfies the equation $\mathrm{SM}^{*} f=J(X)$.

Proof. It is easily verified that

$$
U(X, 0)=\left(\omega_{n-1} r^{n-2}\right)^{-1} \int_{e} J(Y)|Y|^{-1} d Y,
$$

where $2 \rho=r \lambda, Y=r \alpha / 2+r\left(\lambda^{2}-1\right)^{1 / 2} \alpha^{\prime} / 2, X=r \alpha,\left(\lambda^{2}-1\right)^{(n-3) / 2} d \lambda=\rho^{-1}(2 S / r)^{n-2} d S$, $S=2^{-1} r\left(\lambda^{2}-1\right)^{1 / 2}, e$ is the hyperplane perpendicular to $\alpha$ and passing through $X / 2$. If we let $J_{0}\left(X,|X|^{2} / 2\right)=\int_{e} J(Y)|Y|^{-1} d Y$, it follows from the lemma, (24), (29) that

$$
\mathrm{SM}^{*} r \Delta_{X}^{(n-1) / 2} r^{n-2} U(X, 0)
$$

$$
\begin{aligned}
& =2 r\left(\omega_{n-1} \omega_{n}\right)^{-1} \Delta_{X}^{(n-1) / 2} \int_{\alpha^{\prime}} J_{0}\left(X+r \alpha^{\prime},\left|X+r \alpha^{\prime}\right|^{2} / 2\right) d \omega_{\alpha^{\prime}} \\
& =(-1)^{(n-1) / 2}(n-2) ! 2 J(X) .
\end{aligned}
$$

Applying SM* to (21), we find

$$
\mathrm{SM}^{*} r \Delta_{X}^{(n-1) / 2} r^{n-2} U(X, 0)=(-1)^{(n-1) / 2}(n-2) ! 2 \mathrm{SM}^{*} f .
$$

It now follows from (30) that the left-hand side of (31) is equal to

$$
(-1)^{(n-1) / 2}(n-2) ! 2 J(X) .
$$

\section{REFERENCES}

1. Y. W. Chen, On the solutions of the wave equation in the exterior of the characteristic cones, J. Math. Mech. 16 (1967), 655-673. MR 35 \#3290.

2. F. John, Plane waves and spherical means applied to partial differential equations, Interscience, New York, 1955. MR 17, 746.

3. - Bestimmung einer Funktion aus ihren Integralen über gewisse Mannigfaltigkeiten, Math. Ann. 109 (1934), 488-520.

4. $\mathrm{H}$. Rhee, Inversion of the functional equation $\mathrm{SM}^{*} f=J$ in odd-dimensional spaces, Thesis, Univ. of Massachusetts, Amherst, 1968.

State University College of New York, ONEONTA, NEW YoRK 13820 